\title{
APLICACION DE LA VACUNA MIXTA Y RESULTADOS OBTENIDOS
}

\author{
Por el Dr. CONRADO RISTORI C y Stra MARLA VRLALOBOS $M$.
}

Entre todas las enfermedades infecto-contagiosas de la infancia, lä tos ferina representa sin duda el problema epidemiológico más grave, comparable sólo con la tuberculosis por su alta cifra de mortalidad.

La estadística registrada en Chile por la Dirección General de Sanidad revela que! hasta los 5 años, mueren anualmente de bronconeumonía consecutiva a la tos ferina alrededor de 800 niños, itúmero que se leva en los periodos epidémicos de la afección a 1,200 ó más. Si se agrega a lo ante. rior el hecho universalmente conocido de quel es esta la enfermedad infecciosa que con más frecuencia se omite declarar, existiendo una alta proporción de niños que ni siquiera reciben atención médica durante toda su evolución, no es de extrañar que un porcentaje aito de los casos que aparecen registrados como fallecidos de bronconeumonía corresponden a coqueluches en que ella apareció como complicación. En otras palabras, la elstadística. a pesar de la alta cifra de mortalidad que registra, no es in reflejo fiel de la realidad epidemiológica de esta grave en'fermedad de la infancia.

Esto justifica los ésfuerzos realizados por el Instituto Bacteriológico, secundado por la Oficina de Profilaxis de la Dirección General de Sanidad, en orden a obtener un procedimiento de inmunización activa eficiente para evițat o atenuar este problema, y que culminaron con la preparación de la vacuna cuyas caracteristicas fueron expuestas en la presentación anterior.

Con esta vacuna hemos realizado nuestras experiencias a partir de eneiro de 1943 , prevío control minucioso de sù inocuidad en el niño, en atención a su alta concentración microbiana. 
Realizamos esta primera fase de nuestras experiencias en el Hogar Santa Rosa, escogiendo para las primeras inyecciones a los niños mayores, cuya edad fluctuaba entre 5 y 6 años. Fuimos aplicando en grupos similares una cantidad creciente del antígeno, empezando por 1/4 dea c.c. hasta llegar a la dosis definitiva de $1 \mathrm{c}^{\prime} \mathrm{c}$. y observando en cada caso en forma detenida las reacciones locales y generales que' se producían. Establecida de esta manera la ausencia de reacciones de consideración fuimos tomando grupos cada vez menores hasta llegar a lactantes de 8 meses, que consideramos como la edad límite inferior, ya que por debajo de ella el niño parecel no tener aún completamente cesarrollada la facultad de elaborar anticuerpos contra la tos ferina.

Del este modo quedó de manifiesto que la vacuna con la que realizaríamos nuestras experiencias posteriores no daba reacciones de importancia que impidieran su aplicación.

- Las reacciones locales y genetales producidas serán esta. blecidas más adelante.

Vacunación. - Para el ensayo de esta vacuna hemos escogido grupos de niños bien controlados, con el antecetdente preciso de no haber tenido anteriormente la enfermedad, pertenetientes a diversas instituciones de protección a la infancia que existen en el pais, cada uno de los cuales posee una ficha personal y está sometido a examen médico periódico. Entre estas instítuciones, debemos mencionar a la Casa de Socotro de Puente Alto a cuyo Director. Dr. Cantuarias, corresponde buena parte de mérito de esta comunicación: al Consultor:o del Seguro Obrero de la Comuna de San Miguel: a la Maternidad Madre e Hijo; a la Institución Sweet; el Centro Preventivo N." 21 del H. Barros Luco; a la Unidadi Sanitaria de Ouinta Normal; a la Casa de la Madre (perteneciente al Consejo de Defensa del Niño) ; a la Protectora de la Infancia; a la población de Seweel: a la Escuela de Puericultura, $\forall$ por último a la Colocación familiar de la Casa Nacional del Niño.

Todos los vacunados tenían una edad que fluctuaba entre 8 meses y 5 a 6 años, habiéndose ya anotado más attás la razón por la que no se escogieron niños menores. El limite máximo establecido se debió a que por encima de los 6 años la coqueluche deja de ser un problema epidemiológico y clínico de importancia.

No se vacunaron niños que tuvieran antecedentes de tuberculosis. lúes, distrofia, trastornos nutritivos agudos o afecciones cutáneas infecciosas. 
Técnica de la vacunación. - Hemos aplicado nuestra vacuna en dos dosis de 1 c.c. por vía subcutánea, con un mes de intervalo, cambiando de brazo por persistir después de ese lapso en un cierto número de casos una induración discreta en el punto de aplicación de la primera dosis.

En algunos niños hemos distanciado más la apícación de ambas dosis, llegando hasta 3 ó 4 meses, por haber establecido alguinos autores que el ideal para obtener la mayor inmunidad es esperar la total reabsorción del nódulo antes de inyectar una nueva cantidad de antígeno, manten éndose de esta manera por más tiempo el estímulo aplicado.

Hemos, además, evitado el emp'eo de cualquier otra vacura hasta un perríodo de 3 meses después de colocada la segunda dosis.

Con esta técnica hemos procedido a vacunar la mayor cantidad posible de niños, dejando grupos similares de control: de igual edad, pertenecientes al mismo sector, a la misma clase social y, en muchos casos, a la misma familia.

Nos hemos éncontrado con grandes dificultades, especialmente en lo que se refiere a la aplicación de la segunda dosis, debido en gran parte al descuido de los familiäres para concurrir en la fecha indicada.

Reacciones locales y generales. - Hemos estudiado las reacciones locales en 731 inyelcciones, dividién Jolas para su anotación en 4 grupos: Se consideró reacción local positiva con una cruz $(+)$ la caracterizada por la aparición de una pequeña zona indurada en el punto de la inyección. sin cambio de coloración 'de la piel y sin dolor manifiesto. Esta reacc'ón pasó en general inadvertida para los famíliares. $S ?$ anotó como positiva con dos cruces $(++)$ aquella reacción en que el nớdulo inđurado subcutắneo, tenia un diámetro inferior a $2 \mathrm{I} / 2 \mathrm{~cm}$., pero en que aparekía un entojecimiento de la piel en la región correspondiente. Se indicó como positiva con tres cruces $(+++)$ una reacción análoga a la anterior, pero de mayor diámetro.

Por último, en un cierto número de casos se produjo una zona fluctuante correspondiente a pequeños abscesos asépticos ocasionados por la alúmina y que se presentan también con otros preparados aue la contienen. La aparición de estoś abscesos que sólo por excepción se abren espontáneamente al exterior, ya ha sito indicada por los diversos autores americanos que trabajan con este tipo de vacunas, a pesar de la menor concentración microbiana por ellos empleada. 
Por lo que se refiere a las reacciones generales, más escasas que las anteriores, fueron estudiadas en 453 niños. Se limitaron por lo general a un discreto aumento de la temperatura, no acompañado de otros síntomas que demuestreln 'a existencia de un estado febril. Los casos en que aparecieron algunos de ellos, cono anorexia, deca:miento, cambios del carácter, vómitos, etc., son del todo excepcionales.

En general, las reacciones fueron mayores mientras mayor era el niño vacunado, fenómeno ya observado con la aplicación del toxoide diftético sólo y que justifica el que no hayamos utilizado dosis proporcionalmente menores en los lactantes.

A continuación se expone en un cuadro la proporción. de cada una de las reacciones indicadas que se observaron en los niños controlados:

REACCION LOCAL: INYECCЮNES CONTROLADAS, 731

\begin{tabular}{ccc}
\hline Positiva + & Positiva ++ & Positiva +++ \\
$286(39.1 \%)$ & $278(38.0 \%)$ & $167(22.9 \%)$ \\
\hline
\end{tabular}

REACCION GENERAL: CASOS CONTROLADOS, 453

$\begin{array}{ccc}\text { Negativa } & \text { Febricula } & \text { Fiebre } \\ 321(70,9 \%) & 90(19,9 \%) & 42(9,2 \%)\end{array}$

No hemos incluido en el cuadro anterior, los abscesos que presentaron algunos niños, por cuanto los 14 casos en que se observó esta reacción corresponden al total del los vacunados, es decir a 2,958 inyecciones. Como se ha dicho anteriormente, estos abscesos, de contenido asépt co, se deben a la presencia de alúmina como substancia extraña al organismo $y$ en su mayor parte se reabsorbieton espontáneamente, sin cejar alteraciones locales. Seis de ellos se abrieron al exterior, dando salida en 3 casos a un pus espeso, de aspecto cremoso. Es necesario dejar constancia que uno del ellos debe ser considerado más bien como un flegmón del brazo producido por infección accídental en el momento de la inyeccion, habiendo logrado aislarse del pus un estreptococo hemolítico. Otro caso, correspondiente como $e^{1}$ anterior a la Casa de Socorro de Puente Alto, se debió a la vacunación de 
un niño en que ésta estaba contraindicada por presentat una. piodermitis en evolución.

Por lo tanto, él número total de abscesos abiertos espontáneamente a través de la piel, úntco accidente molesto de la vacunación, se limita a 4 sobre un total de 2,958 inyecciones, cifra que está por debajo de las consignadas en trabajos extranjeros y que no supera a los accidentes habituales producidos por cualquier otro tipo de vacuna.

En todos estos casos se logró una rápida restitución a la normalidad, quedando sólo una pequeña cicatriz, similar a la producida por la vacunación antivariólica.

Debemos hacer notar que dos de estos abscesos se presentaron en el mismo caso, con cada una de las dos dosis, por lo que parece existir una predisposición individual. $\mathrm{Se}$ trataba por lo demás de una niña de 7 años, lo que confirma la aselveración anterior de que la reacción local aumenta con la edad.

En resumen, si tomamos en consideración el alto nú. mero de niños vacunados y la ausencia absoluta de accidentes graves, podemos concluir que las reacciones producidas no significan de ninguna manera una contraindicación para este nuevo procedimiento de inqmunización contra dos enfermedades que causan anualmentel un número tan alto de víctimas.

Control epidemiológico. - Un trabajo experimental de esta naturaleza sólo puede llegar a conclusiones convincentes, si se basa en el estudio epidemiológico posterior de los niños vacunados y su comparación con grupos de control.

Una investigación de esta clase requiere una cifra elevada de casos con el objeto de que los resultados obtenidos no puedan ser considerados por la bioestadística como fruto exclusivo del azar.

Hemos vacunado con la primera dosis una cifra total de 1.738 niños y dé estos sólo hemos podido aplicar la segunda en 1,220, debiendo considerarse varios factores como causa de esta diferencia. En primer lugar, muchos niños cambiaron de domicilio siendo imposible ubicarlos nuevamentel En otros casos hubo resistencia por parte de los familiares; en alganos apareció la tos ferina pocos días después de la aplicación de la primera dosis, es decir antes de dar tiempo al organismo para elevar su inmunidad; por último, algunas enfermedades intercurrentes, especialmente trastornos nutritivos agudos tan frecuentes durante el verano, nos obligaron a postergar la aplicación de la segunda dosis o a abandonar el 
caso, cuando el petíodo de separación entre ambas era excésivo.

En algunas de las institucionets a las que hemos recurrido para la ejecución de este trabajo, como la Casa de Socorro de Puente Alto, el Consultorio de San Miguel de la Caja de Seguro Obligatorio; el Centro Preventivo N.? 21 del H. Barros Luco y la Unidad Sanitaria de Quinta Normal, sólo se vacunó una parte de los niños quedanḍo por lo tanto de control grupos similares en lo que se, refiere a edad, medio, social, etc. En otras, por e' contrario (Maternidad Madre e Hijo Institución Sweet), todos los niños fueron inmunizados $y$ por lo tanto sólo podemos recurrir como cifras comparativas a la motbilidad general de Santiago entre las edades consideradas o a la de los Consultorios de la Caja de Serguro Obligatorio de ubicación más próxima a estas instituciones.

Este estudio estadistico comparativo se vió favorecido por la circunstancia casual de haberse realizado gran parte det trabajo dutante el año 1943, que fué excepcionalmente epidémico para esta afección.

Por otra parte, con el objeto de obtener resultados lo más exactos posibles, hemos efectuado este estudio en sectores diversos de la ciudad, muy ale.jados unos de otros, $y$ aun fuera de ella (Sewell, Puente Alto), evitando de esta manera

- Ios errores que podrían resultar de una irregular repartición de los brotes epidémicos en los distintos barrios.

A continuación vamos a exponer en algunos cuadros los resultados estadísticos comparativos entre los grupos de niños vacunados y los controles en cada una de las instituciones.indicadas:

Número de vacunados $\mathbf{s}$ de controles en las diversas instituciones.

\begin{tabular}{|c|c|c|c|c|c|}
\hline & Vacunados & Ent. & Control & Enf. & $\%$ \\
\hline Puente Alto & 358 & 2 & 800 & 137 & 17,1 \\
\hline Inst. Sweet - & $\begin{aligned} 88 \\
280\end{aligned}$ & $\overline{1}$ & - & - & - \\
\hline Preventivo N.9 21 & $\begin{array}{r}269 \\
78\end{array}$ & $\underline{1}$ & $\overline{150}$ & $\overline{48}$ & $\overline{32}$ \\
\hline C. San Miguel . & 265 & - & 605 & 102 & 16,8 \\
\hline U. sanitarla Q. Normal & 74 & 一 & 1,608 & 109 & 6,7 \\
\hline Dispersos ... . . & 87 & - & - & 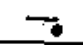 & - \\
\hline TOTAL. & 1.220 & $\begin{array}{c}3 \\
4 \%\end{array}$ & 3,163 & 386 & 12,5 \\
\hline
\end{tabular}


Correcioión del cuadro anterior sezún el periodo de observación de los casos.

\begin{tabular}{|c|c|c|}
\hline & Vacunados & Oontroles \\
\hline 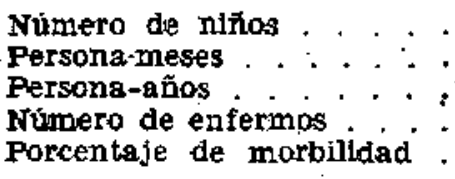 & $\begin{array}{c}1,2,20 \\
11,188 \\
932,33 \\
3 \\
0,32 \%\end{array}$ & $\begin{array}{c}3,162 \\
53,+11 \\
4,480,02 \\
396 \\
8,83 \%\end{array}$ \\
\hline
\end{tabular}

En el primer cuadró puede verse luego la enorme diferencia que existe entre las cifras de morbilidad de los vacunados y de los controles. Mientras en estos últimos ésta fluctuó entre el $6,7 y$ el $32 \%$. con ur términó medio del $12.52 \%$, en los primeros sólo llegó al $0.24 \%$ (3 casos en total). Dos de estos riños pertenecer a la Casa de Socorro de Puente Alto y el tercero a la Maternidad Madre e Hijo; en los 3 casos la enfermedad siguió un curso muy benigno y la evolución fué corta, en uno de ellos de 18 días en total. En ninguno de los 3 fué posible investigar la presencia del $\mathrm{He}^{-}$ mophilus Pertussis en la expectoración, por haber llegado 2 nuestro conocimiento cuando ya, este examer es nelgativo en un porcentaje demasiado elevado de casos para que pueda concedérsele valor.

En el segundo cuadro se reduce la cifra de niños controlados, tanto entre los que recibieron la vacuna como entre los que quedaron de control al número de meses de observación de cada caso, ya que no tiene el mismo valor un control de 17 meses, correspondiente a los primetos casos y uno de 1 mes, como en los últimos. Con la corraceión de estas cifras se llega a los resultadoe anotados en el cuadro. y que son algo inferiores a los establecidos en EE. UU. por P. Kendrick, en un estudio similar.

No hemos considerado aquí los casos de coqueluche aparecidos después de la primera dosis ya que la mayor parte de ellos se produjeron muy pocos dias después de esta aplicación, no dando tempo por tanto al' organismo para elaborat sus anticuerpos.

- Aunque el número total de casos presentados no es muy elevado si se considera la complejidad de un estudio estadístico que se refiere a un programa de inmunización en que tantos factores diversos elntran en juego, la diferencia entre las cifras de morbilidad de los niños vacunados en forma completa y los controles es tan enorme, que no puede dadarse de su valor. 
Exposición directa al contagio. - Es un hecho ya establecido por la experiencia y por la lógica, que la forma más demostrativa de controlar la inmunidad contra una enfermedad infecto-contagiosa es la observación de los casos expuettos accidental o voluntariamente al contagio. Los resultados estadísticos, para no merecer objeciones, deben estar apoyados en cifras enormes, y en un período de control muy prolongado, que están generalmente fuera de las posibilidades de un estudio experimental.

La circunstancia casual ya anotada más atrás. đe haberse practicado la mayor parte del trabajo durante el año 1943, el más intensamenter epidémico pata la tos ferina en los últimos tiempos, permitió que el contagio accidental viniera a apoyat nuestras conclusiones. Hemos reunido una cifra aptoximada de 60 casos que se mantuvieron en contacto íntimo intrafamiliar con niños afectados por esta enfermedad, siendo los únicos que escaparon a la infección.

Creemos de interés exponer por separado algunas de estas obseryaciones que nos parecen concluyentes.

En la Casa de la Madre, perteneciente al Consejo de Defensa del Niño, sel mantiene constantemente un número aproximado de 20 de ellos en tránsito, es decir, por espacio de algunos meses, hasta que pueden ser entregados a sus familiares. Eni enero de 1943 procedimos a vacunar a todos los asilados que no habían hecho anter:ormentel la tos ferina, pudiendo esto set constatado en la ficha personal de cada uno de elloss. Dos meses después de colocada la 'seygunda dosis, y en condiciones que sólo 5 de los niños de esta experiencia permanecían aún en la institución, habiendo sido los demás reemplazados por otros que no fueton vacunados, apareció un brote epidémico de la enfermedad que abartó a los 9 recién ingresados, respetando ún ccamente a los 5 inmunizados. Debemos hacer presente que algunos de estos niños habian permanecido en el establecimiento casi desde su naci. miento, existiendo por tarto la absoluta seguridad de que no habian enfermado anteriormentel.

En el Jardín Infantil de la Casa de Socorro de Puente Alto se produjo una situación análoga a la anterior. En un niño de $3 \pi / 2$ años que sólo había recibico la primera dosis de la vacuna 3 idias antes, apareció la coqueluche, que sólo pudo diagnostícarse 6 días después, permaneciendo por tanto durante todo ese tiempo con 18 compañeros todos vacunados. ninguno de los cuales enfermó. Esté grupo inclaye una hermana dell niño, que permaneció en contacto intrafamiliar con él durante toda la evolución de la enfermedad. De la expec- 
toración de este caso pudo ser aislado el Hemophilus Pertussis un the's después cie iniciado el cuadro clínico.

En una familia domiciliada en la Comuna de San Miguel, cuyos niños estaban inscritos en el Centro Preventivo N." 21 (H. Barros Luco), fueron vacunados los dos de edad intermedia, quedando de control al mayor y el menor, de 6 años y 8 meses. respectivamente. La tos ferina afectó posteriormente a estos últimos, respetando a los dos primeros.

En ofra familia, de Puente Alto, en que sólo uno de los 6 niños que pertenecen a ella fué vacunado, enfermaron los 5 restantes, escapando a la infección el niño inmunizado, a pesar de' no baber sido aislado de sus hermanos durante toda la evolución de la enfermedad de ellos. Este caso es aún más demostrativo si se considera que se trataba del hijo menor, que sólo contaba con 10 meses de edad en el momento del contagio y que, por tanto debe ser considerado el más receptivo.

Casos idénticos a los expuestos se suceden hasta tom- pletar la cifra de 60 indicada más arriba, estando todos registrados por separados en nuestras anotaciones. Entre los niños no vacunados expuestos al contagio la morbilidad sería, según trabajos americanos, entre el 70 y $90 \%$.

Creemos que estas observaciones son del más alto valor en lo que a estel procedimiento de inmunización se refiere y constituyen el argumento más importante a favor del mismo. que podemos presentar.

Control secológico. - Hemos creido de interés completar nuestro estudio sobre este nuevo procedimiento de vacunación, practicando en 150 casos reacciones de aglutinación un mes después de colocada la segunda dosis. Hemos preferido este control serológico a otros utilizados por diversos investigadores, entre los cuales se cuentan la desviación del complemento, el test de protección a la laúcha, el estudio del. poder opsónico, etc., por tratarse de un método más sencillo que los otros y de resultados más o menos concordantes con ellos.

Como esta parte del trabajo se encuentra aún inconclusa, preferimos no referirnos a ella en esta comunicación, pudiendo sí anticipar que en los primeros 60 casos estas han sido positivas en 57 a títulos diversos, debiéndose recordar a elste propósito que normalmente no existen aglutininas contra el Hemophilus pertussis, ni aun a bajo título. Para controlar nuestro antígeno hemos practicado en 14 casos las reacciones citadas antes de la vacunación, siendo negativas en todos ellos. 
Como pueden haber apreciado los que escuchan esta presentación, hemos dado una atención preferente en esta vacuna mixta, a la patte que se refiera a la tos ferina, no preocupándonos de la inmunización antidiftérica. Lo hemos hecho deliberadamente por considerar esta segunda fase del problema como ya resuelta no habiendo en este momento dis cordancia entre los autores en lo que se refiere a la protección antidiftérica conferida por el toxoide aluminado en las dosis habituales de $30 \mathrm{Lf}$, repetidas con un mes de intervalo, dosis que como ya se dejó establecido, es la que contiène la vacuna que nos ocupa, a pesar de esto, existielndo un medio fácil y seguro de establecer la inmunidad antidiftérica, como es la reacción de Schick, se completó este trabajo practicando dicha relacción en 150 casos, un mes después de la aplicación de la segunda dosis. Entre estos hemos tenido 7 resaltados positivos, lo que da un porcentaje de negatividad del $95.3 \%$, mientras en niños de la misma edad no inmunizados este varía alrededor del $30 \%$.

\section{Conclusiones.}

1. El empleo de una vacuna mixta anti-pertussis diftérica precipitada en alúmina y de alta concentración microbiana $(40,000$ millones de hermophilus pertussis por c.c.) demostró conferir una elevada inmunidad a los niños en los cuajes se aplicó.

2." No se han observado con esta vacuna reacciones locales o generales mayores que las descritas por los autores norteamericanos con las que ellos atilizan y en las cuales la concentración microbiana es la mitad o la cuarta parte que la indicada.

3. - La edad más adecuada para el empleo de esta vacuna parece estar comprendida entre los 8 meses y los 5 años, debiendo ser la dosis empleada la misma para las diversas edades, no existiendo indicación para fraccionarla en los lactantes ya que la reacción local es mayor mientras mayor es el niño.

4." No puede : establecerse en este trabajo la duración de la inmunidad, por cuanto sólo se inició un año y medio antes de esta comunicación. Tampoco existe experiencia extranjera sobre este punto.

5.9 Estimamos que la vacuna mixta aluminada antidiftérica-pertussis en dos dosis, debe ser agregada a los procedimientos rutinarios de inmunización activa actualmente ntilizados por las instituciones encargadas del control de la Higiene Pública. 